\title{
Nanostructural Deformation Analysis of Calcium Silicate Hydrate in Portland Cement Paste by Atomic Pair Distribution Function
}

\author{
Hiroshi Suzuki, ${ }^{1}$ Sungchul Bae, ${ }^{2}$ and Manabu Kanematsu ${ }^{3}$ \\ ${ }^{1}$ Materials Sciences Research Center, Japan Atomic Energy Agency, 2-4 Shirakata, Tokai, Naka, Ibaraki 319-1195, Japan \\ ${ }^{2}$ Division of Architectural Engineering, Hanyang University, Seoul 04763, Republic of Korea \\ ${ }^{3}$ Department of Architecture and Building Engineering, Faculty of Science and Technology, Tokyo University of Science, \\ 2641 Yamazaki, Noda, Chiba 278-8510, Japan \\ Correspondence should be addressed to Sungchul Bae; sbae@hanyang.ac.kr
}

Received 15 November 2015; Accepted 3 March 2016

Academic Editor: Jozef Bednarcik

Copyright (C) 2016 Hiroshi Suzuki et al. This is an open access article distributed under the Creative Commons Attribution License, which permits unrestricted use, distribution, and reproduction in any medium, provided the original work is properly cited.

\begin{abstract}
The deformation of nanostructure of calcium silicate hydrate (C-S-H) in Portland cement (PC) paste under compression was characterized by the atomic pair distribution function (PDF), measured using synchrotron X-ray diffraction. The PDF of the PC paste exhibited a unique deformation behavior for a short-range order below $2.0 \mathrm{~nm}$, close to the size of the C-S-H globule, while the deformation for a long-range order was similar to that of a calcium hydroxide phase measured by Bragg peak shift. The compressive deformation of the C-S-H nanostructure was comprised of three stages with different interactions between globules. This behavior would originate from the granular nature of $\mathrm{C}-\mathrm{S}-\mathrm{H}$, which deforms with increasing packing density by slipping the interfaces between globules, rearranging the overall C-S-H nanostructure. This new approach will lead to increasing applications of the PDF technique to understand the deformation mechanism of C-S-H in PC-based materials.
\end{abstract}

\section{Introduction}

Portland cement (PC) paste is composed of various hydration products, such as calcium hydroxide $(\mathrm{CH})$, ettringite, monosulphate, and calcium silicate hydrate (C-S-H), as well as several anhydrous $\mathrm{PC}$ clinker phases [1]. C-S-H, a primary binding phase in $\mathrm{PC}$ paste, has predominant influences on its mechanical and chemical properties. It is, therefore, crucial to understand the role of the C-S-H phase in the deformation behavior of PC paste.

In previous studies, various discussions on the mesoscale structure of C-S-H have been made regarding the basis of physical properties such as specific surface area, pore size, and density measured by a number of observation techniques [2-4]. Jennings, accordingly, suggested that the basic model for the nanostructure of C-S-H to represent these physical parameters, which is a particle-packing model with nanoscale globules, is known as the colloid model $[5,6]$. This is a useful model for understanding the physical and mechanical properties of C-S-H as in the case of Constantinides and Ulm [7] discovering the unique nanogranular behavior of C-S-H, driven by particle-to-particle contact forces, from the results of the mapping of the indentation modulus.

Although there are various deformation studies on PC paste on the basis of $\mathrm{CH}$ phase behavior measured using neutron and X-ray diffraction techniques [8-10], there is a lack of studies on the deformation of C-S-H itself due to its unique nanostructure. Meanwhile, the atomic pair distribution function (PDF) technique is well known as a method to evaluate nanoscale structures of materials with no or less crystal periodicity. For instance, using the PDF technique, Skinner et al. suggested that the size of the C-S-H nanoparticle is estimated to be approximately $3.5 \mathrm{~nm}$ in diameter [11]. Furthermore, this technique has been successfully applied to quantitative evaluation of the deformation behavior of amorphous metallic glasses by directly measuring displacement between 


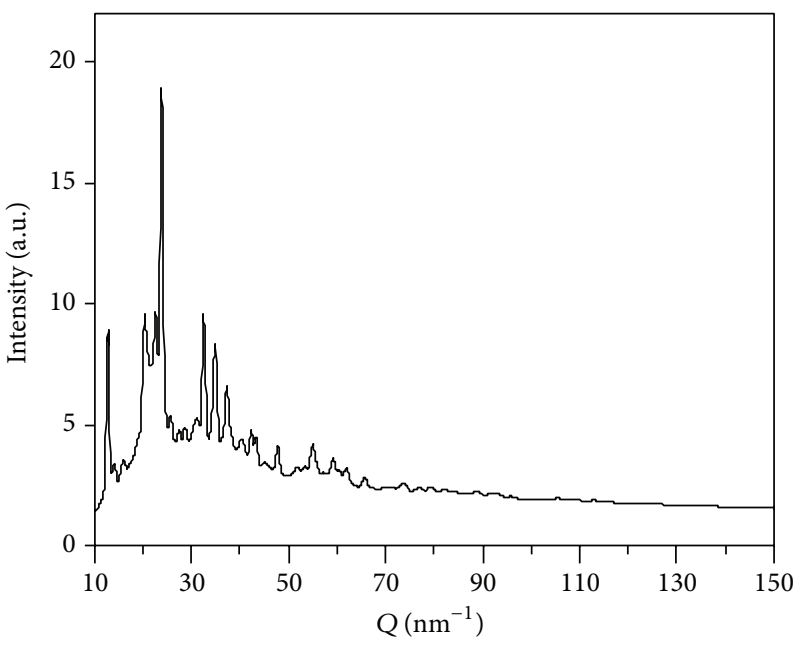

(a)

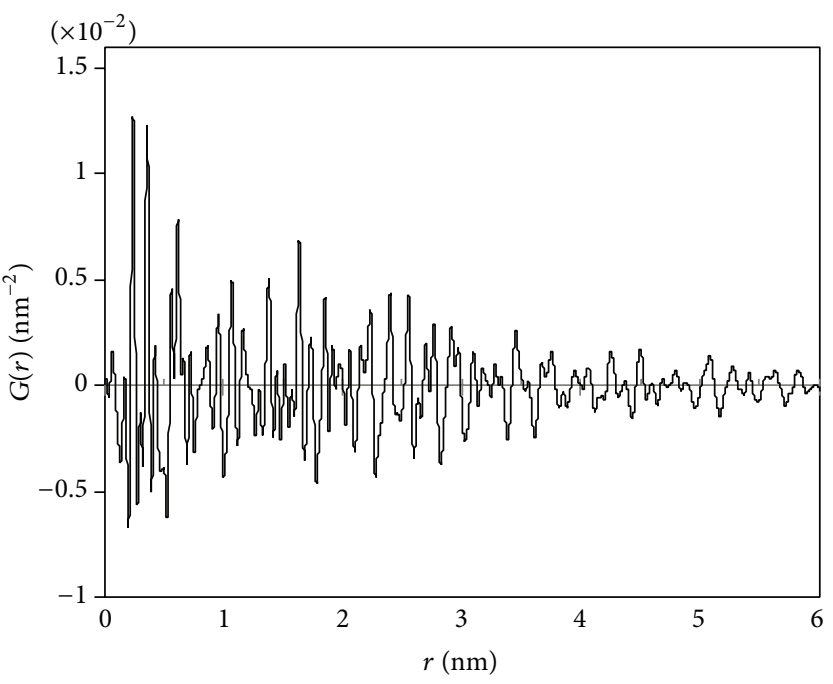

(b)

Figure 1: (a) X-ray diffraction pattern of PC paste measured at the detector position of $300 \mathrm{~mm}$ and (b) the PDF data derived from the diffraction pattern shown in (a).

neighbor atoms [12]. In this study, the deformation behavior of the $\mathrm{C}-\mathrm{S}-\mathrm{H}$ phase in PC paste is discussed by applying the PDF technique to access a quantitative evaluation of the local strains occurring in the $\mathrm{C}-\mathrm{S}-\mathrm{H}$ nanostructure.

\section{Material and Methods}

PC for research containing fewer impurities was mixed with a water-to-cement ratio of 0.5 . The paste was cured for a total of 73 days, that is, demolded after 24 hours, cured in water for 28 days, and stored for 45 days under ambient conditions at $20^{\circ} \mathrm{C}$ and $60 \% \mathrm{RH}$. The rectangular specimen, with a dimension of $5 \times 5 \times 10 \mathrm{~mm}^{3}$, was obtained from the PC paste with dimensions of $50 \times 50 \times 92 \mathrm{~mm}^{3}$.

The X-ray diffraction experiments were carried out using high-energy X-rays of $69.8 \mathrm{keV}$ at BL22XU in SPring-8, Japan [13]. The specimen mounted on the load frame was set on the diffractometer and was irradiated by an incident beam with a size of $0.3 \times 0.3 \mathrm{~mm}^{2}$. The diffraction from the specimen was measured by an imaging plate (IP) with exposure time of 20 seconds. The pixel size of the IP was approximately $0.1 \mathrm{~mm}$. The distances from the IP to the specimen were set to be $300 \mathrm{~mm}$ and $700 \mathrm{~mm}$. Each distance was determined to obtain a longer Q-range for the PDF and higher angular resolution for the strain analysis by Bragg peak shift, respectively. A diffraction pattern in the loading direction was obtained by circumferentially integrating the range of $\pm 30^{\circ}$ of the diffraction ring. The compressive loadings were applied to the specimen with the constant displacement mode until approximately $40 \mathrm{MPa}$. Since the applied stress was gradually released due to creep while stopping the displacement, the average stress during measurement was defined to be applied stress. The scattering vector was parallel to the axis of the applied compressive load.

\section{Results}

Figure 1(a) presents a diffraction pattern measured by the IP placed at $300 \mathrm{~mm}$. A number of diffraction peaks can be observed, which are derived from hydration products and anhydrous clinker phases. Although most diffraction peaks are difficult to identify because of their complex crystal structures, $\mathrm{CH}$ peaks can be extracted from the diffraction pattern since it is a simple trigonal structure. In contrast, the $\mathrm{C}-\mathrm{S}-\mathrm{H}$ nanostructure might appear as a halo pattern overlapped with the diffraction pattern. Figure 1(b) shows the $\mathrm{PDF}$ produced by the PDFgetX3 program [14] with a $Q$-range of Fourier transformation from 14 to $140 \mathrm{~nm}^{-1}$. The radius, $r$, in the horizontal axis in Figure 1(b) is the distance from an average atom located at the origin. This is mixed nanostructural information of all composed phases, including C-S-H, weighted by the volume fraction, and shows similar trends regarding the PDF of the hydrated tricalcium silicate paste shown in the previous study [11].

Figure 2 shows a change in strains as a function of the applied stress, derived from the Bragg peak shift. The elastic strain, $\varepsilon_{b}$, is obtained by a change in the lattice spacing, $d$, for $h k l$ reflection in the sample from reference lattice spacing, $d_{n 0}$, in an initial step of loading; $\varepsilon_{b}=\left(d_{n}-d_{n 0}\right) / d_{n 0}$. The deformation of the $\mathrm{CH}$ phase (hereafter "CH-strain") was calculated by averaging strains of 36 reflections related to the $\mathrm{CH}$ phase using the following equation:

$$
\varepsilon_{b_{\text {Avg }}}=\frac{1}{n} \sum \varepsilon_{b_{n}}=\frac{1}{n} \sum \frac{d_{n}-d_{n 0}}{d_{n 0}}
$$

where $n$ indicates the number of reflections used for the strain calculation. In contrast, the average deformation of other composed crystalline phases, that is, hydration products except $\mathrm{C}-\mathrm{S}-\mathrm{H}$ and $\mathrm{CH}$ phases, was calculated by averaging the 


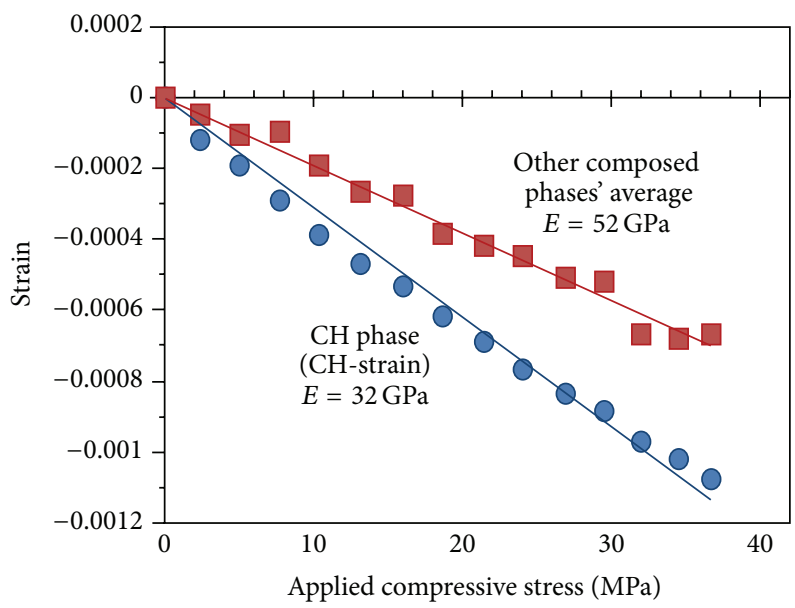

Figure 2: Strain change of the $\mathrm{CH}$ phase and the average strain of the other composed phases as a function of the applied compressive stress. The strains are derived from the Bragg peak shift. The average error bars are $\pm 3.4 \times 10^{-5}$ and $\pm 2.1 \times 10^{-4}$, respectively. The lineally approximated lines are presented in solid lines.

strains of the remaining 22 reflections. The $\mathrm{CH}$ phase shows a linear deformation, but we can see a slight change in gradient at $14 \mathrm{MPa}$ of compressive loading by carefully looking. Assuming it is linear, Young's modulus of the $\mathrm{CH}$ phase is calculated to be approximately $32 \mathrm{GPa}$, which is smaller than that of other composed phases, approximately $52 \mathrm{GPa}$.

The PDF shown in Figure 1(b) represents a mixed atomic distribution derived from all composed phases including $\mathrm{C}-\mathrm{S}-\mathrm{H}$, weighted by the volume fraction of each phase. It is, therefore, challenging to extract only C-S-H structural information from the measured PDF. In this study, we attempted to characterize the deformation behavior of C-S$\mathrm{H}$ by using the feature that the intensity damping of the PDF is correlated to the damping of the structural coherence in the crystalline grain, meaning the grain size [15]. The PDF in the short range could be dominated by C-S-H since its volume fraction is typically known to be approximately $50 \%$ or more [16]. In contrast, the structural information of C-S-H in the PDF decreases with an increase of $r$ since the discrete PDF derived from $\mathrm{C}-\mathrm{S}-\mathrm{H}$ with the size of a few nanometers might be damping immediately.

In the PDF technique, the atomic strain, $\varepsilon_{a}$, is obtained by a change of the peak position, $r$, from a reference radius, $r_{n 0}$, in an initial step of loading; $\varepsilon_{a}=\left(r_{n}-r_{n 0}\right) / r_{n 0}$. Figure 3 shows the atomic strain behaviors that averaged at intervals of $0.5 \mathrm{~nm}$ or $1.0 \mathrm{~nm}$ in $r$, calculated by the following equation:

$$
\varepsilon_{a_{\mathrm{Avg}}}=\frac{1}{n} \sum \varepsilon_{a_{n}}=\frac{1}{n} \sum \frac{r_{n}-r_{n 0}}{r_{n 0}}
$$

where $n$ indicates the number of peaks used for the strain calculation. The change in the atomic strain in the short range below $1.0 \mathrm{~nm}$ shows the S-curve and shifts the trend to be linear gradually with an increase in $r$. With exceeding $2.0 \mathrm{~nm}$ in $r$, the atomic strains change linearly along the $\mathrm{CH}$-strain measured by the Bragg peak shift but do not always agree with the $\mathrm{CH}$-strain beyond $3.0 \mathrm{~nm}$ in $r$. According to the result shown in Figure 3, $2.0 \mathrm{~nm}$ in the PDF seems to be a critical size in the transition of deformation. Considering the typical globule size of C-S-H, $\sim 5.0 \mathrm{~nm}$, suggested by various techniques in previous studies $[4-6,11,17]$, the deformation behavior below $2.0 \mathrm{~nm}$ in $r$ might be predominantly characterized by the $\mathrm{C}-\mathrm{S}-\mathrm{H}$ nanostructure. Figure 4 shows a change in atomic strains averaged in the ranges from $0 \mathrm{~nm}$ to $2.0 \mathrm{~nm}$ and from $2.0 \mathrm{~nm}$ to $6.0 \mathrm{~nm}$, compared with the $\mathrm{CH}$-strain. In the local structural deformation below $2.0 \mathrm{~nm}$ in $r$, the plateau region can be observed from 14 to $24 \mathrm{MPa}$ of applied stress in compression. On the other hand, the deformation in the longer range between $2.0 \mathrm{~nm}$ and $6.0 \mathrm{~nm}$ shows a slightly similar trend to the local structural deformation, but it rather corresponds to the $\mathrm{CH}$-strain. This means that the deformation above $2.0 \mathrm{~nm}$ in $r$ predominantly represents the deformation of the $\mathrm{CH}$ phase.

\section{Discussion}

As shown in Figure 4, the deformation behavior of the shortrange structure in the range from $0 \mathrm{~nm}$ to $2 \mathrm{~nm}$ would consist of three stages with different trends as follows. The linear deformation behavior with $10 \mathrm{GPa}$ of Young's modulus is observed first in Stage-I, and then the deformation becomes smaller in Stage-II. After that, the compressive strain linearly increases again with $37 \mathrm{GPa}$ of Young's modulus in Stage-III. Here, it is appropriate to apply the colloid model $[5,6]$ to explain the unique deformation behavior of the short-range structure within $2.0 \mathrm{~nm}$ in $r$ on the basis of the nanogranular nature.

Figure 5 shows the schematic idea regarding the deformation mechanism of C-S-H under compression. Assuming that C-S-H acts as a granular material, the force transmission between globules follows the principle of the force chain network [18]. Considering an initial structure of C-S-H in StageI, low-density C-S-H with large gel pores decreases Young's modulus since the small contact area, that is, the small loadbearing area, between globules enhances deformation in C-S$H$. Furthermore, there would be no slip between globules in Stage-I. In Stage-II, on the other hand, the interface filled 


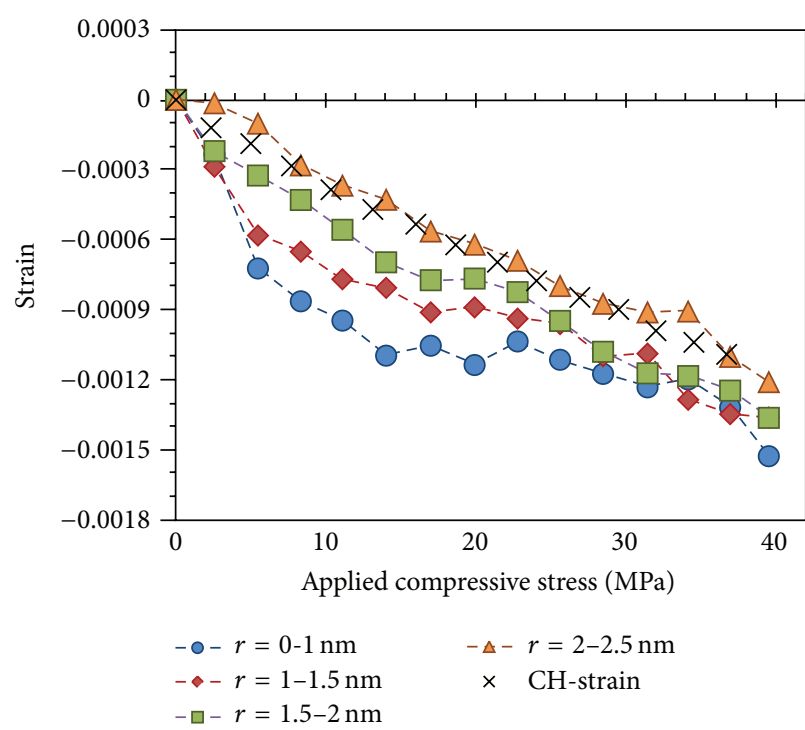

(a)

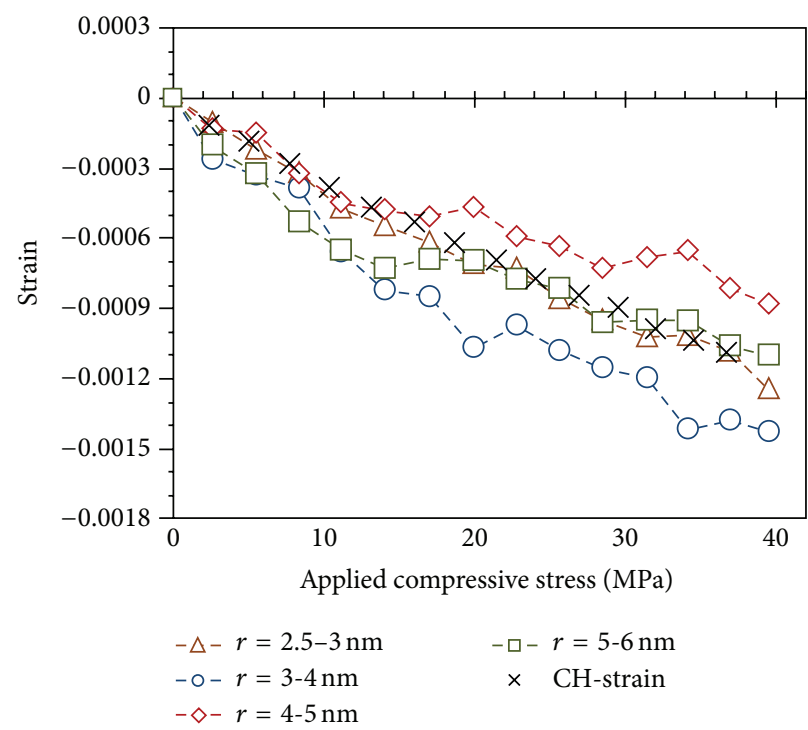

(b)

Figure 3: The atomic strains that averaged at intervals of $0.5 \mathrm{~nm}$ or $1.0 \mathrm{~nm}$ in $r$, compared with the strain of the $\mathrm{CH}$ phase derived from the Bragg peak shift. The average error bars are $\pm 22 \times 10^{-5}(r=0-1 \mathrm{~nm}), \pm 9.5 \times 10^{-5}(r=1-1.5 \mathrm{~nm}), \pm 6.1 \times 10^{-5}(r=1.5-2 \mathrm{~nm}), \pm 6.3 \times 10^{-5}(r=$ $2-2.5 \mathrm{~nm}), \pm 4.0 \times 10^{-5}(r=2.5-3 \mathrm{~nm}), \pm 3.2 \times 10^{-5}(r=3-4 \mathrm{~nm}), \pm 2.5 \times 10^{-5}(r=4-5 \mathrm{~nm})$, and $\pm 1.8 \times 10^{-5}(r=5-6 \mathrm{~nm})$.

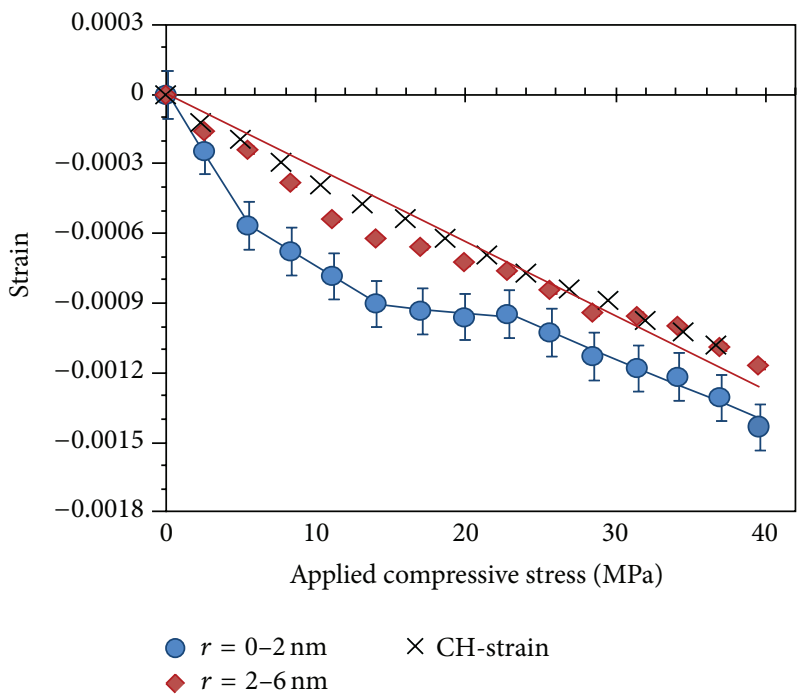

FIGURE 4: Change in the atomic strains that averaged in the ranges from $0 \mathrm{~nm}$ to $2.0 \mathrm{~nm}$ in $r$ and from $2.0 \mathrm{~nm}$ to $6.0 \mathrm{~nm}$ in $r$, compared with a change in the strain of the $\mathrm{CH}$ phase derived from the Bragg peak shift. The average error bars are $\pm 1.0 \times 10^{-4}$ and $\pm 1.5 \times 10^{-5}$, respectively.

by cohesive water between globules might start to slip since shear stress exceeds the critical strength of the interface, resulting in an increase in the packing density in C-S-H and then starting inhomogeneous deformation. While increasing the packing density in Stage-II, Young's modulus in C-S-H is physically increasing with an increase in the load-bearing area between globules, and the strain in C-S-H decreases by following Hooke's law even if there is no change in the applied loading. This implies that the strain in C-S-H can be relaxed by physical increasing in Young's modulus of C-S-H during deformation. In the first half of Stage-II, a partial slip occurs between globules in the C-S-H structure, and Young's modulus slightly increases with an increase of the solid-phase density. Following an increase in the applied stress, a slip occurs all over the C-S-H structure, resulting in a large increase in Young's modulus and apparently showing small increase in strain, a plateau, in the second half of Stage-II. After full packing of globules in C-S-H, the C-S-H phase deforms homogeneously in Stage-III with $37 \mathrm{GPa}$ of Young's modulus. The difference in Young's modulus between Stage-I and StageII indicates changes in the nanostructure, with an increase in density and a decrease in gel pores in $\mathrm{C}-\mathrm{S}-\mathrm{H}$ by compressive deformation. In accordance with the reference [7], the selfconsistent model indicates that the indentation modulus of C-S-H can also increase more than threefold by an increase in the packing density of about $20 \%$. Therefore, it is no wonder that this large difference in Young's modulus appears between Stage-I and Stage-III.

\section{Conclusions}

In this study, the deformation behavior of the C-S-H nanostructure was quantitatively characterized by the PDF technique, taking the feature of intensity damping in the PDF, which is correlated to the damping of the structural coherence in the crystalline grain size. The compressive deformation of the C-S-H nanostructure would consist of three stages with different interactions between globules. It would originate from the granular nature of C-S-H which deforms with increasing the packing density by slipping the interlayer between globules, rearranging the overall C-S-H nanostructure. However, it is necessary to isolate specific atom-atom 


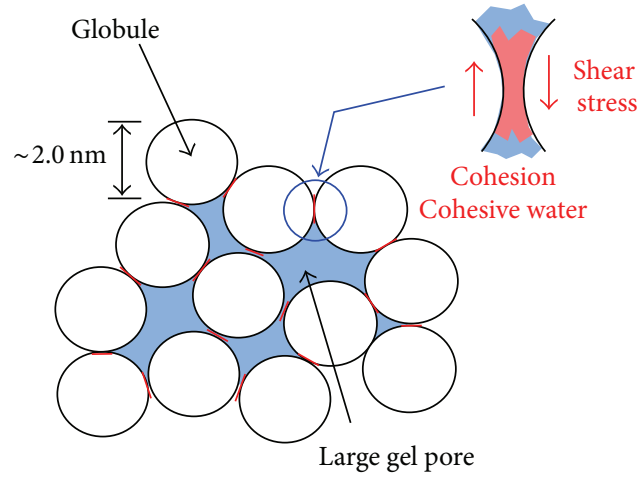

Stage-I (0-6 MPa)

(i) Elastic deformation (no slip)

(ii) Low-density C-S-H

(iii) Lower Young's modulus (10 GPa)
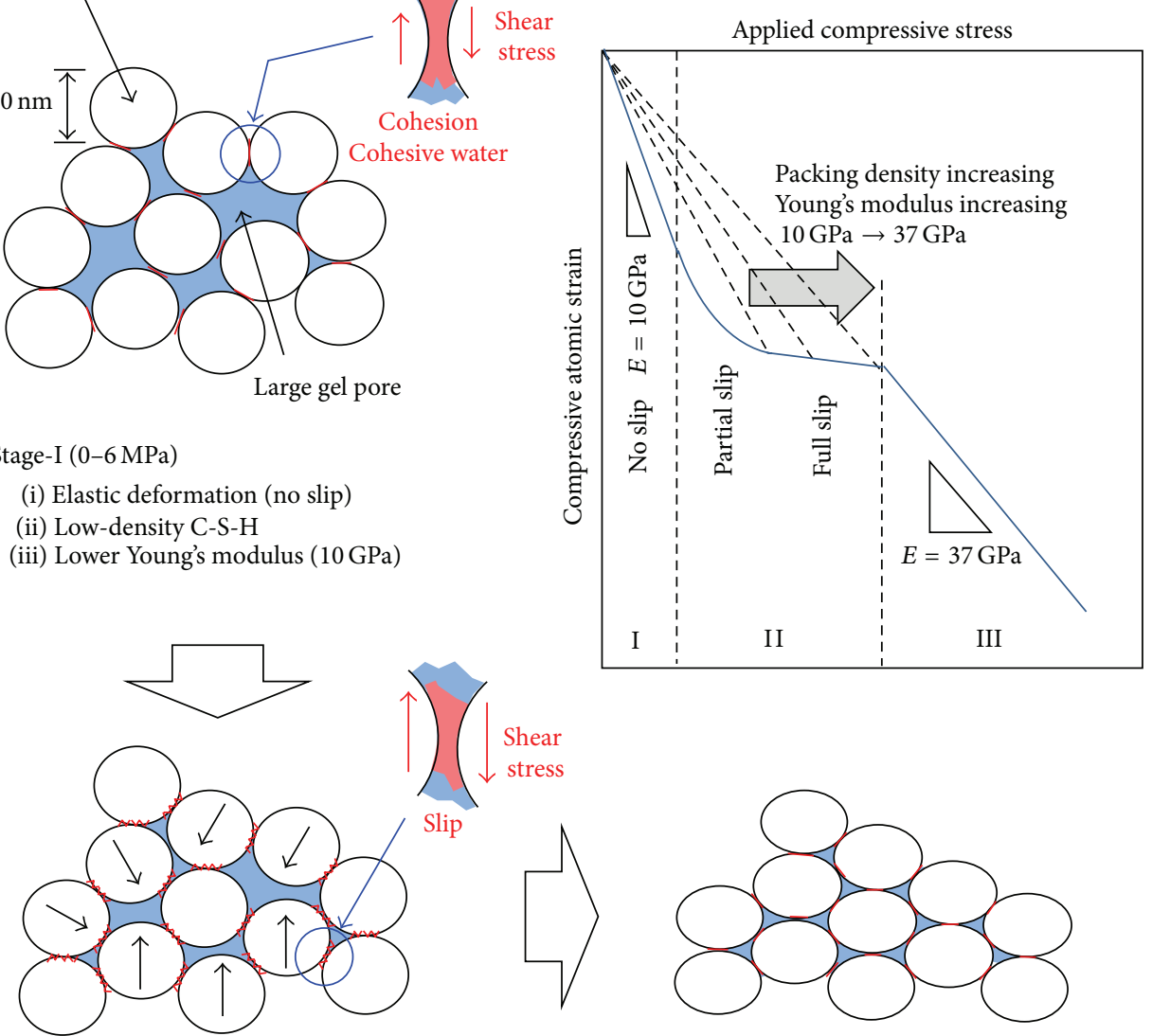

Stage-II (6-23 MPa)
(i) Inhomogeneous deformation (slip)
(ii) Move globules closer
(iii) Packing density increasing
(iv) Young's modulus physically increasing
Stage-III (23-40 MPa)
(i) Homogeneous elastic deformation
(ii) High density C-S-H
(iii) Higher Young's modulus (37 GPa)

Figure 5: Schematic illustration of the deformation mechanism of C-S-H under uniaxial compression. Arrows in the globules in Stage-II indicate an image of its movement.

correlations due to the $\mathrm{C}-\mathrm{S}-\mathrm{H}$ nanostructure throughout $\mathrm{PDF}$ data, since the PDF data presented in this study contain the contribution of other hydrated and anhydrous crystalline phases. Careful modeling of PDF using a pure PC clinker phase paste could enable us to remove the effect of other phases on PDF data. This more detailed understanding enables the C-S-H deformation model in PC paste, as well as PC-based systems containing supplementary cementitious materials.

\section{Competing Interests}

The authors declare that they have no competing interests.

\section{Acknowledgments}

The synchrotron radiation experiment was performed at the SPring-8 with the approval of the Japan Synchrotron Radiation Research Institute (JASRI) as Proposal nos. 2013B3724,
2014A3724, and 2014B3724. This work was supported by the research fund of Hanyang University (HY-2016). The authors wish to acknowledge the experimental assistance of Drs. T. Watanuki, A. Machida, T. Shobu, and A. Shiro at the Japan Atomic Energy Agency (JAEA); Professor M. Imafuku, Mrs. K. Shimizu, and S. Tsubaki at Tokyo City University; and Mr. S. Shiroishi at Tokyo University of Science. The authors would also like to acknowledge Dr. K. D. Liss at the Australian Nuclear Science and Technology Organisation and Drs. N. Igawa and K. Kodama at JAEA for their beneficial assistance.

\section{References}

[1] J. W. Bullard, H. M. Jennings, R. A. Livingston et al., "Mechanisms of cement hydration," Cement and Concrete Research, vol. 41, no. 12, pp. 1208-1223, 2011.

[2] J. J. Thomas, H. M. Jennings, and A. J. Allen, "The surface area of hardened cement paste as measured by various techniques," Concrete Science and Engineering, vol. 1, pp. 45-64, 1999. 
[3] W. P. Halperin, J.-Y. Jehng, and Y.-Q. Song, "Application of spinspin relaxation to measurement of surface area and pore size distributions in a hydrating cement paste," Magnetic Resonance Imaging, vol. 12, no. 2, pp. 169-173, 1994.

[4] A. J. Allen, J. J. Thomas, and H. M. Jennings, "Composition and density of nanoscale calcium-silicate-hydrate in cement," Nature Materials, vol. 6, no. 4, pp. 311-316, 2007.

[5] H. M. Jennings, "A model for the microstructure of calcium silicate hydrate in cement paste," Cement and Concrete Research, vol. 30, no. 1, pp. 101-116, 2000.

[6] H. M. Jennings, "Refinements to colloid model of C-S-H in cement: CM-II," Cement and Concrete Research, vol. 38, no. 3, pp. 275-289, 2008.

[7] G. Constantinides and F.-J. Ulm, "The nanogranular nature of C-S-H," Journal of the Mechanics and Physics of Solids, vol. 55, no. 1, pp. 64-90, 2007.

[8] S. E. Mikel, J. J. Biernacki, and T. Gnapel-Herold, "A neutron diffraction-based technique for determining phase resolved strains in Portland cement," ACI Journal, vol. 106, pp. 455-460, 2009.

[9] J. J. Biernacki, C. J. Parnham, T. R. Watkins, C. R. Hubbard, and J. Bai, "Phase-resolved strain measurements in hydrated ordinary portland cement using synchrotron X-rays," Journal of the American Ceramic Society, vol. 89, no. 9, pp. 2853-2859, 2006.

[10] E. M. Schulson, I. P. Swainson, and T. M. Holden, "Internal stress within hardened cement paste induced through thermal mismatch: calcium hydroxide versus calcium silicate hydrate," Cement and Concrete Research, vol. 31, no. 12, pp. 1785-1791, 2001.

[11] L. B. Skinner, S. R. Chae, C. J. Benmore, H. R. Wenk, and P. J. M. Monteiro, "Nanostructure of calcium silicate hydrates in cements," Physical Review Letters, vol. 104, no. 19, Article ID 195502, 2010.

[12] H. F. Poulsen, J. A. Wert, J. Neuefeind, V. Honkimäki, and M. Daymond, "Measuring strain distributions in amorphous materials," Nature Materials, vol. 4, no. 1, pp. 33-36, 2005.

[13] T. Watanuki, A. MacHida, T. Ikeda et al., "Development of a single-crystal X-ray diffraction system for hydrostatic-pressure and low-temperature structural measurement and its application to the phase study of quasicrystals," Philosophical Magazine, vol. 87, no. 18-21, pp. 2905-2911, 2007.

[14] P. Juhás, T. Davis, C. L. Farrow, and S. J. L. Billinge, "PDFgetX3: a rapid and highly automatable program for processing powder diffraction data into total scattering pair distribution functions," Journal of Applied Crystallography, vol. 46, no. 2, pp. 560-566, 2013.

[15] K. Kodama, S. Iikubo, T. Taguchi, and S.-I. Shamoto, "Finite size effects of nanoparticles on the atomic pair distribution functions," Acta Crystallographica Section A, vol. 62, no. 6, pp. 444-453, 2006.

[16] D. P. Bentz, "Three-dimensional computer simulation of portland cement hydration and microstructure development," Journal of the American Ceramic Society, vol. 80, no. 1, pp. 3-21, 1997.

[17] Z. Xu and D. Viehland, "Observation of mesostructure in calcium silicate hydrate gels of portlad cement," Physical Review Letters, vol. 77, pp. 952-955, 1996.

[18] J. F. Peters, M. Muthuswamy, J. Wibowo, and A. Tordesillas, "Characterization of force chains in granular material," Physical Review E, vol. 72, no. 4, Article ID 041307, 2005. 

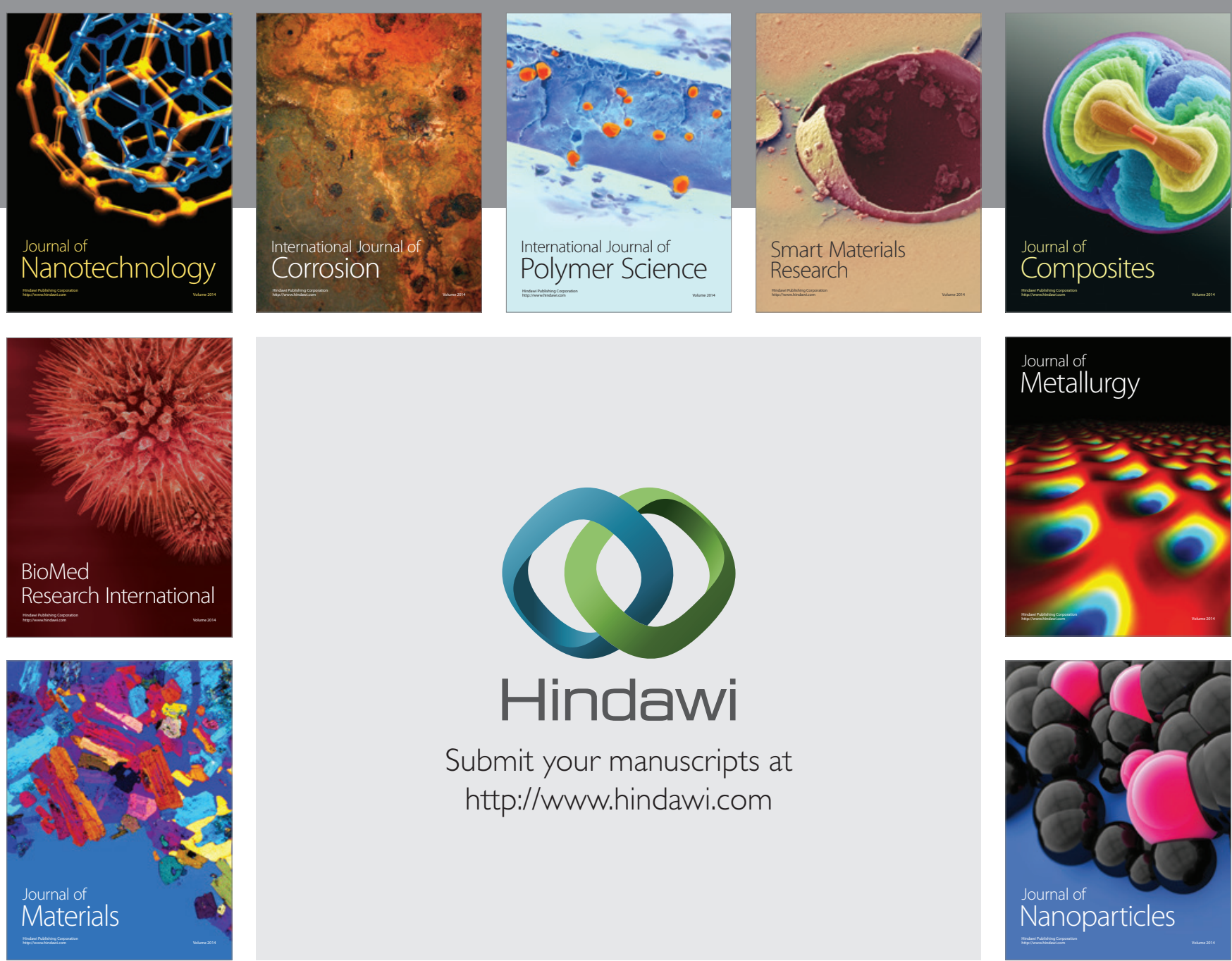

\section{Hindawi}

Submit your manuscripts at

http://www.hindawi.com

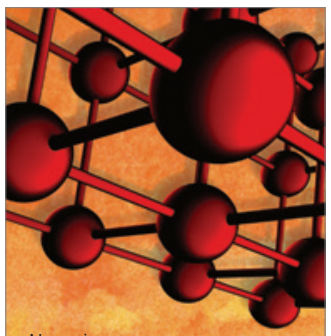

Materials Science and Engineering
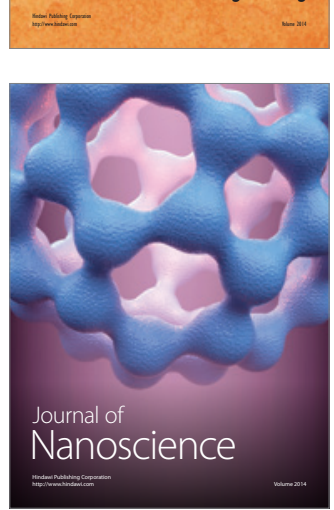
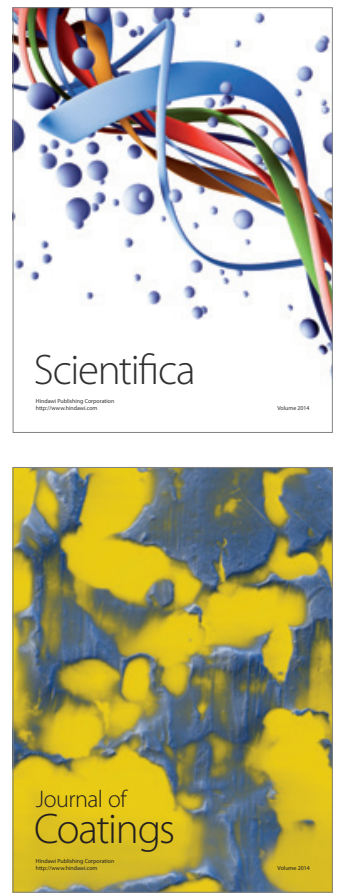
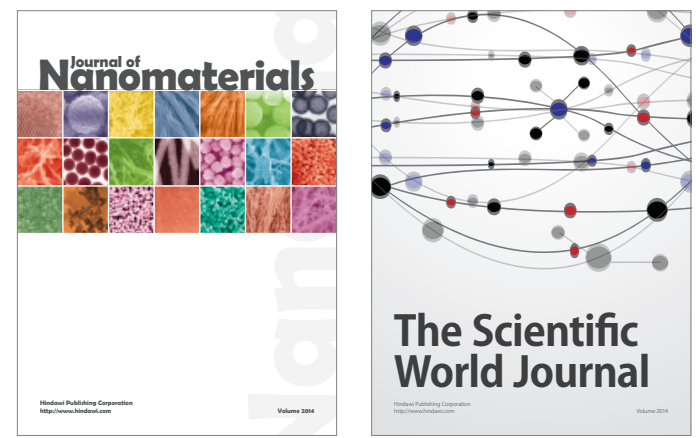

The Scientific World Journal
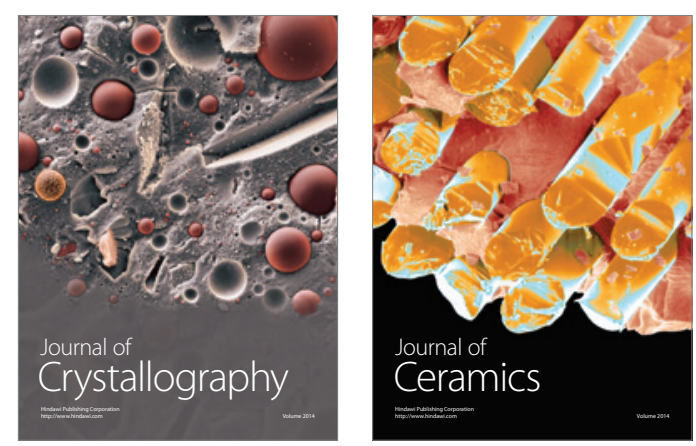
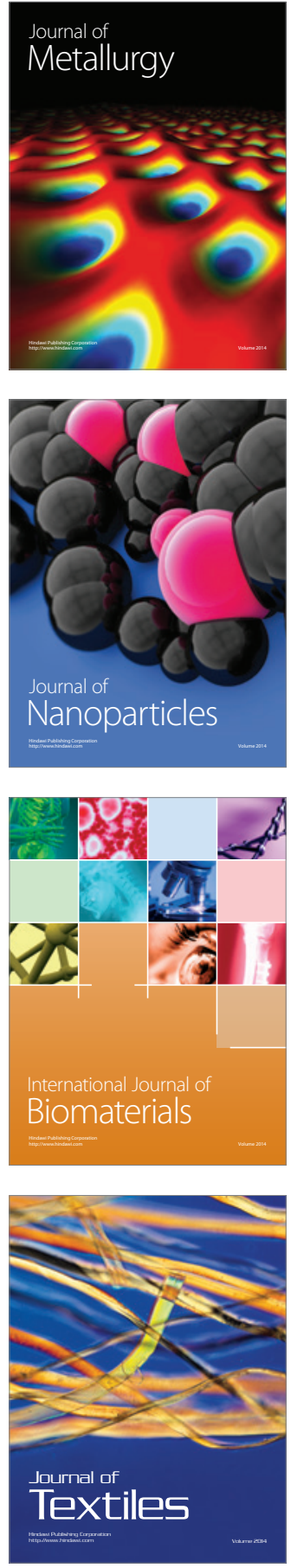